\title{
AVALIAÇÃO DE MODELOS NA SECAGEM DO RESIDUO DE MARACUJA EM SECADOR ROTATÓRIO COM LEITO FIXO ADAPTADO
}

\author{
B. D. de MOURA ${ }^{1}$, E. L. de OLIVEIRA ${ }^{2}$, F. C. MORAES Filho ${ }^{3}$ e J. A. de OLIVEIRA ${ }^{4}$
}

${ }^{1}$ Universidade Federal do Rio Grande do Norte

${ }^{2}$ Universidade Federal do Rio Grande do Norte, Departamento de Engenharia Química

${ }^{3}$ Universidade Federal do Rio Grande do Norte

${ }^{4}$ Universidade Federal do Rio Grande do Norte, Departamento de Engenharia Química

E-mail para contato: briggidadantas@yahoo.com.br

\begin{abstract}
RESUMO: Com o crescimento da agroindústria e crescente demanda nas indústrias de processamento de polpa de frutas, veio a necessidade de encontrar formas de aproveitamento para os resíduos, transformando-os em benefícios financeiros e minimizando impactos ambientais. A secagem destes resíduos, além de agregar valor a um novo produto, possibilita o manuseio, transporte e armazenamento sem perdas significativas. O secador rotatório é um dos sistemas que pode ser utilizado na secagem desses resíduos das indústrias de processamento. Este trabalho teve por objetivo estudar a automação de um secador rotatório, implementando um sistema de aquisição de dados para o controle e monitoramento da temperatura do ar na alimentação, e avaliar o comportamento da secagem convectiva do resíduo do maracujá nas temperaturas de $75^{\circ} \mathrm{C}$ e $95^{\circ} \mathrm{C}$, utilizando os modelos de Henderson e Pabis, Lewis, Page, Page Modificado, Fick Simplificado, Yagcioglu et al., Midilli e Kucuck e Vermas et al.
\end{abstract}

\section{INTRODUÇÃO}

A operação de secagem objetiva sempre a qualidade do produto final. A importância do processo de secagem para as indústrias é no sentido do beneficiamento e qualidade do produto, redução de custos do armazenamento, transporte e prolongamento da vida útil do produto na prateleira. Os secadores rotatórios podem apresentar diferentes comprimentos, diâmetros e forma de alimentação do material sólido em contato com o gás. Esses equipamentos são classificados quanto à forma de transferência de calor em: secadores de contato direto e indireto. No primeiro caso o gás de secagem entra em contato direto com o material em processamento e no segundo caso o contato de ambos ocorre por meio de uma superfície aquecida indiretamente.

Um secador rotatório convencional é constituído por um cilindro que gira mediante suportes apropriados, normalmente com pouca inclinação em relação à horizontal e o comprimento do cilindro podendo variar de quatro a dez vezes o seu próprio diâmetro que pode medir de 0,3 m a mais de 3 metros. (PERRY, 1999). Nesse secador, a alimentação é 
realizada na extremidade do cilindro e se movimenta, em concorrente ou contracorrente em relação ao gás de secagem, até a outra extremidade. Geralmente, peças suspensores internas, conhecidas como fligths, deslocam o sólido e põem em contato mais eficiente com a corrente de gás (Vieira e Silveira, 1995).

Apesar da importância dos processos de secagem com secadores rotatórios para as indústrias, o controle automático com estes sistemas é ainda bastante precário, já que muitas instalações são geralmente conduzidas ainda em modo de operação manual. Basicamente, o processo de secagem numa instalação rotatória consiste na redução da umidade de um produto até um valor desejado. Desta forma, o alvo principal de controle num secador rotatório é estabelecer a umidade do produto na saída do equipamento.

Para a determinação das variáveis que caracterizam o processo de secagem, assim como a obtenção de um maior domínio e conhecimento nos fundamentos dos mecanismos envolvidos, muitos pesquisadores tem estudado a secagem de alimentos em camada fina.

Existem métodos empíricos, teóricos e semiteóricos para descrever matematicamente o processo de secagem. O método empírico geralmente se baseia nas condições externas de secagem, não fornecendo indicações sobre o transporte de energia e massa no interior do produto, muito embora forneça informações práticas para elaboração de projetos (Fortes e Okos, 1980). Diversos modelos matemáticos têm sido utilizados para descrever o processo de secagem de produtos da agroindústria. Embora várias teorias tenham sido propostas para predizer o comportamento da secagem, na maioria das vezes, as expressões semiempíricas e empíricas têm-se mostrado como opções para predizer o processo de secagem, apesar de sua validade estar restrita às condições sob as quais os dados experimentais foram obtidos (Brooker et al., 1992). Este trabalho tem como objetivo estudar vários modelos matemáticos semiempíricos na secagem de resíduo de maracujá.

\section{MATÉRIAS E MÉTODOS}

A matéria prima utilizada neste trabalho, resíduo de maracujá, foi obtida na indústria de beneficiamento de polpa de frutas "Delicia da Fruta" localizada em Natal RN.

O secador rotatório utilizado para secagem do resíduo de maracujá, conforme mostrado na Figura 1 opera com fluxo concorrente ou paralelo e aquecimento direto, de modo que o gás quente (ar) entra em contato com o material a ser processado e ambos se deslocam na mesma direção. $\mathrm{O}$ túnel de secagem possui uma pequena inclinação em relação à horizontal e consiste de um tubo de $60 \mathrm{~cm}$ de comprimento, $15 \mathrm{~cm}$ de diâmetro e 3 suspensores, fixados dentro do túnel, com $3 \mathrm{~mm}$ espessuras e $3 \mathrm{~cm}$ de largura. 


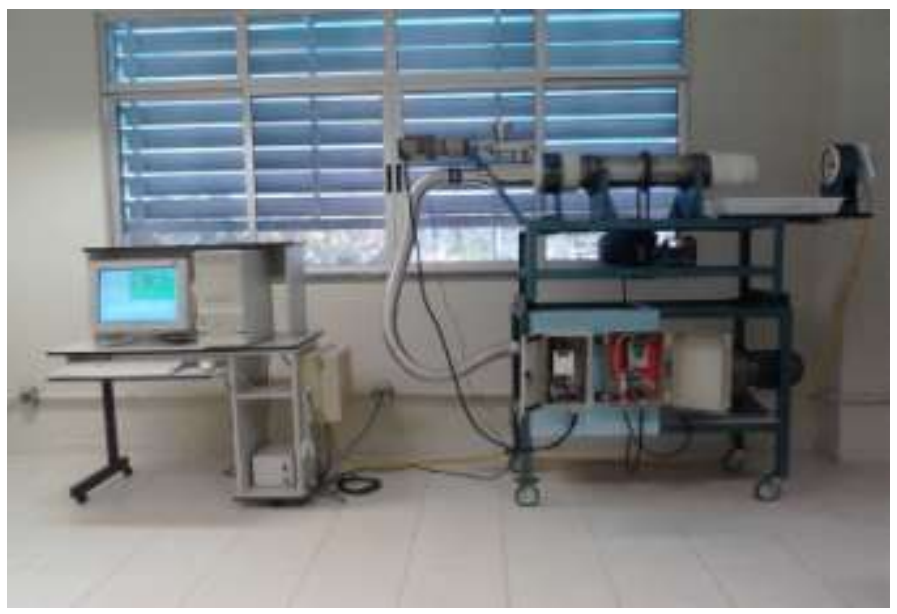

Figura 1 - Secador Rotatório com sistema de controle para secagem de maracujá.

O secador foi adaptado com o objetivo de instalar sensores e elemento final de controle. Os sensores foram instalados no equipamento para medida da temperatura do ar na alimentação e na saída, e umidade do ar na saída do secador. Em relação ao elemento final de controle, utilizou-se de um módulo de potência no qual é possível atuar digitalmente modulando-se a potência da resistência elétrica.

A complexidade do processo durante a secagem conduz pesquisadores a sugerirem varias teorias e fórmulas empíricas para predizer a taxa de secagem. Dentre essas teorias, destaca-se a teoria difusional, que tem como base a Lei de Fick (Equação 1), onde expressa que o fluxo de massa por unidade de área é proporcional ao gradiente de concentração da umidade do material (Akgun e Doymaz, 2005; Park et al., 2003).

$$
\frac{\partial M}{\partial t}=D \frac{\partial^{2} M}{\partial x^{2}}
$$

Onde: "M" é o teor de umidade (kg de água por $\mathrm{kg}$ de sólidos secos), "x" é o deslocamento (m), t é o tempo (s) e "D" é o coeficiente de difusividade, $\left(\mathrm{m}^{2} . \mathrm{s}^{-1}\right)$.

Modelos semiteóricos para secagem em camada fina são, geralmente, derivados da simplificação de uma solução em série da segunda Lei de Fick. É o caso do modelo de Henderson e Pabis que é o primeiro termo de uma solução em série da segunda Lei de Fick (Doymaz, 2005). De acordo com Akpinar (2006), os vários modelos existentes devem ser testados para condições de secagem específicas. Para o presente trabalho foram selecionados os modelos mostrados na Tabela 1 .

Tabela 1 - Modelos Matemáticos semiempiricos

\begin{tabular}{|c|c|c|c|}
\hline Nome Modelo & Equação do Modelo & Equação & Referências \\
\hline Henderson e Pabis & $M R=a \exp (-k t)$ & $(2)$ & Henderson e Pabis (1961) \\
\hline Lewis & $M R=\exp (-k t)$ & $(3)$ & Bruce (1985) \\
\hline Page & $M R=\exp \left(-k t^{n}\right)$ & $(4)$ & Page (1949) \\
\hline
\end{tabular}




\begin{tabular}{|c|c|c|c|}
\hline Page Modificado & $M R=\exp \left(-(k t)^{n}\right)$ & $(5)$ & White et al. (1981) \\
\hline Fick Simplificado & $M R=a \exp \left(-d\left(t / L^{2}\right)\right)$ & $(6)$ & Diamente e Munro (1991) \\
\hline Yagcioglu et al. & $M R=a \exp (-k t)+c$ & $(7)$ & Yagcioglu et al. (1999) \\
\hline Midilli e Kucuck & $M R=a \exp \left(-k t^{n}\right)+b t$ & $(8)$ & Midilli et al. (2002) \\
\hline Vermas et al. & $M R=a \exp (-k t)+(1-a) \exp (-g t)$ & $(9)$ & Vermas et al. (1985) \\
\hline
\end{tabular}

Onde: MR: razão de umidade, adimensional; k, b, c, g: parâmetro de secagem, $\mathrm{s}^{-1}$; n, a, c: parâmetros, adimensional; d: parâmetros de secagem, $\mathrm{m}^{2} \cdot \mathrm{s}^{-1}$; t: tempo, s.

Para determinar a cinética da secagem de resíduo do maracujá, foi indispensável se fazer uma adaptação no secador rotatório de fluxo continuo (Figura 1) de maneira a permitir um sistema em leito fixo. Neste sentido, foi construída uma célula metálica, com $13,7 \mathrm{~cm}$ de diâmetro e $1,5 \mathrm{~cm}$ de espessura, revestida por uma tela onde pudesse colocar o material e manter as condições operacionais da secagem, como mostradas na Figura 2 (a e b).

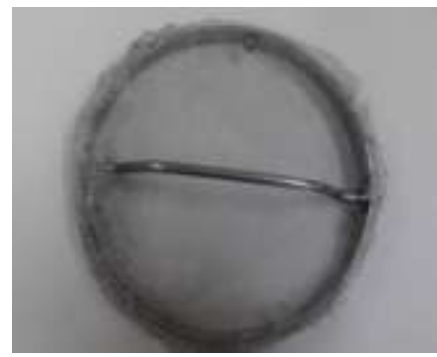

(a)

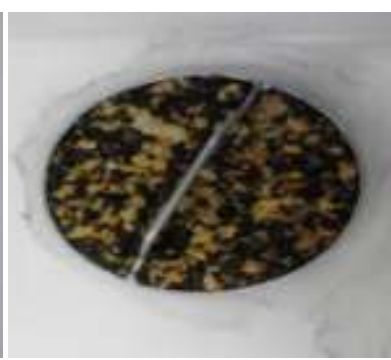

(b)

Figura 2 - Configuração da célula para o leito fixo (a) sem material (b) com material.

Os experimentos, para este trabalho, foram realizados no Laboratório de Energia Alternativa e Fenômenos de Transporte (LEAFT) do Departamento de Engenharia Química da UFRN. O leito fixo teve as seguintes características e condições operacionais: $1,5 \mathrm{~cm}$ de espessura e $170 \mathrm{~g}$ do resíduo de maracujá, vazão do ar de $0,02 \mathrm{~m}^{3} / \mathrm{s}$, temperaturas de $75^{\circ} \mathrm{C}$ e $95^{\circ} \mathrm{C}$. A temperatura de entrada do ar de secagem foi monitorada através de um termopar digital instalado na entrada do secador que foi mantida constante com valor pré-estabelecido (set-point).

Para o estudo cinético do processo, efetuaram-se pesagem do material em processamento em função de tempo pré-estabelecidos. Este procedimento foi repetido até que o material, nessas condições operacionais, obtivesse massa constante. Em seguida, a célula era colocada numa estufa com circulação de ar a $105^{\circ} \mathrm{C}$ por $24 \mathrm{~h}$ para determinar o teor de umidade final do material.

Os dados experimentais obtidos na secagem, em camada delgada, foram ajustados aos modelos citados na Tabela 1 e os parâmetros desses modelos foram obtidos por análise de regressão não linear, utilizando-se o software STATISTICA 7. Para o ajuste dos modelos, utilizou-se o coeficiente de correlação $\left(\mathrm{R}^{2}\right)$ e o parâmetro qui-quadrado reduzido $\left(\chi^{2}\right)$ calculado pela Equação 10. 


$$
\chi^{2}=\frac{\sum_{i=1}^{n}\left(M R_{\text {exp }, i}-M R_{p r e d, i}\right)^{2}}{N-n}
$$

Onde: MRexp,i - valores experimentais da razão de umidade para a observação, i (adimensional); MRpred,i - valores preditos da razão de umidade para a observação, i (adimensional); $\mathrm{N}$ - número de observações; $\mathrm{n}$ - número de parâmetros no modelo para equação 10.

\section{RESULTADOS E DISCUSSÃO}

As Figuras 3 e 4 mostram os resultados da secagem do resíduo de maracujá, nas condições de temperatura $75^{\circ} \mathrm{C} \mathrm{e} 95^{\circ} \mathrm{C}$, respectivamente. As curvas são apresentadas na forma adimensional, umidade final/umidade inicial (Uo/Ui) em função do tempo de secagem, aos modelos propostos de Lewis, Page, Page modificado e Henderson e Pabis (H. P), Fick simplificado, Yagcioglu et al., Midilli e Kucuck e Verma et al.

\section{Temperatura 75으}

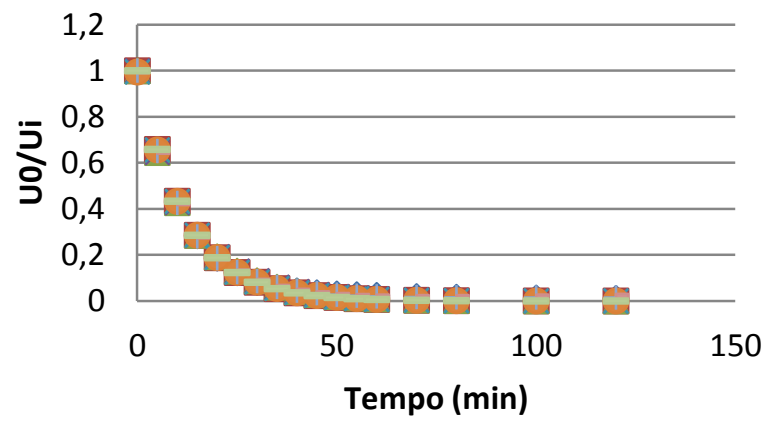

$$
\begin{aligned}
& \text { Experimental } \\
& \text { - Lewis } \\
& \text { × Page } \\
& \text { X H. P. } \\
& \text { - Fick Simplif } \\
& \text { + Yagcioglu } \\
& \text { - Midilli e Kucuck }
\end{aligned}
$$

Figura 3 - Secagem do bagaço do maracujá à temperatura de $75^{\circ} \mathrm{C}$.

Temperatura 95으

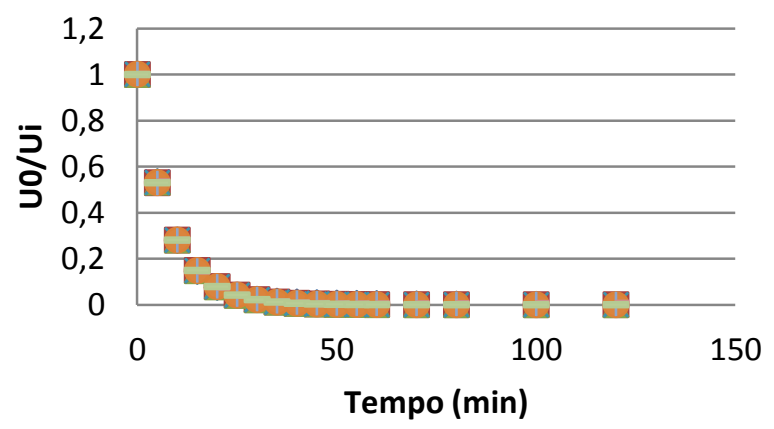

$$
\begin{aligned}
& \text { Experimental } \\
& \text { - Lewis } \\
& \text { ×age } \\
& \times \text { Page mod } \\
& \text { * H. P. } \\
& \text { - Fick Simp } \\
& \text { + Yagcioglu } \\
& \text { - Midilli e Kucuck }
\end{aligned}
$$

Figura 4 - Secagem do bagaço do maracujá à temperatura de $95^{\circ} \mathrm{C}$. 
Avaliando as Figuras 3 e 4, observa-se um comportamento cinético similar de cada curva em relação as condições operacionais utilizadas. Neste caso especifico como o único parâmetro variável foi a temperatura, obviamente o tempo de secagem foi menor com o aumento da temperatura, ou seja, o gráfico da Figura 4 mostra que no período de aproximadamente 25 minutos o resíduo de maracujá já atingiu a umidade constante, enquanto que neste mesmo período no gráfico da Figura 3 o processo ainda continua em andamento e a umidade do resíduo de maracujá tende a ser constante em aproximadamente 50 minutos. Nota-se ainda que todo processo de secagem ocorre no período de taxa decrescente, não observando período de taxa constante o que está de acordo com Ferreira et al. (2012) na secagem do bagaço de uva.

Observa-se também que todos os modelos estudados foram bem ajustados aos dados experimentais, de acordo com os coeficientes de determinação $\mathrm{R}^{2}$ e $\chi^{2}$ apresentados nas Tabelas 2 e 3.

Tabela 2 - Parâmetros dos Modelos à Temperatura $75^{\circ} \mathrm{C}$

\begin{tabular}{|c|c|c|c|c|c|c|}
\hline Modelos & \multicolumn{6}{|c|}{ Parâmetros } \\
\hline \multirow{2}{*}{ Lewis } & \multicolumn{4}{|c|}{$\mathrm{K}$} & $\mathrm{R}^{2}$ & $\chi^{2}$ \\
\hline & \multicolumn{4}{|c|}{0,08362} & 0,99839 & 0,00012 \\
\hline \multirow{2}{*}{ Page } & \multicolumn{2}{|c|}{$\mathrm{k}$} & \multicolumn{2}{|c|}{$\mathrm{n}$} & $\mathrm{R}^{2}$ & $\chi^{2}$ \\
\hline & \multicolumn{2}{|c|}{0,094317} & \multicolumn{2}{|c|}{0,955466} & 0,99872 & 0,0001 \\
\hline \multirow{2}{*}{ Page Modificado } & \multicolumn{2}{|c|}{$\mathrm{k}$} & \multicolumn{2}{|c|}{$\mathrm{n}$} & $\mathrm{R}^{2}$ & $\chi^{2}$ \\
\hline & \multicolumn{2}{|c|}{0,084489} & \multicolumn{2}{|c|}{0,955466} & 0,99873 & 0,0001 \\
\hline \multirow{2}{*}{ Henderson e Pabis } & \multicolumn{2}{|c|}{$\mathrm{a}$} & \multicolumn{2}{|c|}{$\mathrm{k}$} & $\mathrm{R}^{2}$ & $\chi^{2}$ \\
\hline & \multicolumn{2}{|c|}{0,994452} & \multicolumn{2}{|c|}{0,083168} & 0,99843 & 0,00013 \\
\hline \multirow{2}{*}{ Fick Simplificado } & \multicolumn{2}{|c|}{$\mathrm{a}$} & d & $\mathrm{L}$ & $\mathrm{R}^{2}$ & $\chi^{2}$ \\
\hline & \multicolumn{2}{|c|}{0,994452} & 0,08651 & 1,01988 & 0,99843 & 0,00014 \\
\hline \multirow{2}{*}{ Yagcioglu et al. } & \multicolumn{2}{|c|}{$\mathrm{a}$} & $\mathrm{k}$ & $\mathrm{c}$ & $\mathrm{R}^{2}$ & $\chi^{2}$ \\
\hline & \multicolumn{2}{|c|}{0,985711} & 0,0875 & 0,01496 & 0,99987 & 0,00001 \\
\hline \multirow{2}{*}{ Midilli e Kucuck } & $\mathrm{a}$ & $\mathrm{k}$ & $\mathrm{n}$ & $\mathrm{b}$ & $\mathrm{R}^{2}$ & $\chi^{2}$ \\
\hline & 1,00038 & 0,08869 & 0,98404 & 0,00017 & 0,9997 & 0,00003 \\
\hline \multirow{2}{*}{ Verma et. al. } & \multicolumn{2}{|c|}{$\mathrm{a}$} & $\mathrm{k}$ & $\mathrm{g}$ & $\mathrm{R}^{2}$ & $\chi^{2}$ \\
\hline & \multicolumn{2}{|c|}{$-1,33214$} & 0,08329 & 0,08343 & 0,99839 & 0,00014 \\
\hline
\end{tabular}

Tabela 3 - Parâmetros dos Modelos à Temperatura $95^{\circ} \mathrm{C}$

\begin{tabular}{|c|c|c|c|c|}
\hline \multirow{2}{*}{ Modelos } & \multicolumn{4}{|c|}{ Parâmetros } \\
\hline \multirow{2}{*}{ Lewis } & $\mathrm{k}$ & $\mathrm{R}^{2}$ & $\chi^{2}$ \\
\cline { 2 - 5 } & $\mathrm{k}$ & $\mathrm{k}, 127003$ & 0,99977 & 0,00002 \\
\hline \multirow{2}{*}{ Page } & 0,123916 & 1,010609 & 0,99978 & 0,00002 \\
\cline { 2 - 5 } & $\mathrm{k}$ & $\mathrm{n}$ & $\mathrm{R}^{2}$ & $\chi^{2}$ \\
\hline \multirow{2}{*}{ Page Modificado } & 0,126663 & 1,010609 & 0,99978 & 0,00002 \\
\cline { 2 - 5 } & \multicolumn{4}{|c|}{} \\
\hline
\end{tabular}




\begin{tabular}{|c|c|c|c|c|c|c|}
\hline \multirow{2}{*}{ Henderson e Pabis } & \multicolumn{2}{|c|}{$\mathrm{a}$} & \multicolumn{2}{|c|}{$\mathrm{k}$} & $\mathrm{R}^{2}$ & $\chi^{2}$ \\
\hline & \multicolumn{2}{|c|}{1,001379} & \multicolumn{2}{|c|}{0,127158} & 0,99977 & 0,00002 \\
\hline \multirow{2}{*}{ Fick Simplificado } & \multicolumn{2}{|c|}{ a } & $\mathrm{d}$ & $\mathrm{L}$ & $\mathrm{R}^{2}$ & $\chi^{2}$ \\
\hline & \multicolumn{2}{|c|}{1,001379} & 0,122251 & 0,980516 & 0,99977 & 0,00002 \\
\hline \multirow{2}{*}{ Yagcioglu et al. } & \multicolumn{2}{|c|}{$\mathrm{a}$} & $\mathrm{k}$ & $\mathrm{c}$ & $\mathrm{R}^{2}$ & $\chi^{2}$ \\
\hline & \multicolumn{2}{|c|}{0,998865} & 0,128591 & 0,003456 & 0,99988 & 0,00001 \\
\hline \multirow{2}{*}{ Midilli e Kucuck } & $\mathrm{a}$ & $\mathrm{k}$ & $\mathrm{n}$ & $\mathrm{b}$ & $\mathrm{R}^{2}$ & $\chi^{2}$ \\
\hline & 1,000331 & 0,122446 & 1,017202 & 0,00005 & 0,99988 & 0,00001 \\
\hline \multirow{2}{*}{ Verma et. al. } & \multicolumn{2}{|c|}{$\mathrm{a}$} & $\mathrm{k}$ & $\mathrm{g}$ & $\mathrm{R}^{2}$ & $\chi^{2}$ \\
\hline & \multicolumn{2}{|c|}{$-1,00067$} & 0,126943 & 0,126973 & 0,99977 & 0,00002 \\
\hline
\end{tabular}

\section{CONCLUSÕES}

Observou-se que o secador rotatório adaptado para secagem em camada delgada pode ser utilizado para estudar a cinética de secagem do resíduo de maracujá.

Os modelos usados neste trabalho descreveram satisfatoriamente os dados experimentais nas condições operacionais utilizadas, porém os modelos de Yagcioglu et al. e Midilli e Kucuck foram os que melhor ajustaram com $\mathrm{R}^{2}$ mais próximo de um (1) e $\chi^{2}$ mais próximo de zero (0).

De acordo com os resultados obtidos no modelo de Fick simplificado, pode-se verificar que a difusividade aumenta com o aumento da temperatura, para temperatura de $75{ }^{\circ} \mathrm{C} \mathrm{o}$ parâmetro que representa a difusividade (d) foi de $0,08651 \mathrm{~m}^{2} / \mathrm{s}$ e para $95{ }^{\circ} \mathrm{C}$ foi de 0,12225 $\mathrm{m}^{2} / \mathrm{s}$. Pois a difusividade representa a velocidade com que a água migra do interior para a superfície do material, sendo então vaporizada.

Os resultados das Tabelas 2 e 3 mostram que quanto maior a temperatura de trabalho melhor será o comportamento cinético do processo de secagem para o bagaço de maracujá.

\section{REFERÊNCIAS}

AKGUN, N. A.; DOYMAZ, I. Modeling of olive cake thin-layer drying process. Journal of Food Engineering, v. 68, n. 4, p. 455-461, 2005.

AKPINAR, E. K. Determination of suitable thin layer drying curve model for some vegetables and fruits. Journal of Food Engineering, v. 73, n. 1, p. 75-84, 2006.

BROOKER, D. B.; BAKKER-ARKEMA, F.W.; HALL, C.W. Drying and storage of grains and oilseeds. Westport: The AVI Publishing Company, p. 450, 1992. 
BRUCE, D. M. Exposed layer barley drying, three models fitted to new data up to $150^{\circ} \mathrm{C}$. J. Agrip. Eng. Res., v. 32, p. 337-347, 1985.

DIAMENTE, L. M.; MUNRO, P. A. Mathematical modelling of hot air drying of sweet potato slices. Int J Food Sci Technol, 26:99, 1991.

DOYMAZ, I. Drying behaviour of green beans. Journal of Food Engineering, v. 69, n. 2, p. 161-165, 2005.

FERREIRA, L. F. D.; PIROZI, M. R.; RAMOS, A. M; PEREIRA, J. A. M. Modelagem matemática da secagem em camada delgada de bagaço de uva fermentado - Pesq. agropec. bras., Brasília, v.47, n.6, p.855-862, jun. 2012.

FORTES, M.; OKOS, M.R. Drying theories; their bases and limitations as applied to foods and grains. In: MUJUNDAR, A.S. (ed.). Advances in drying. New York: Science Press, 1980. p.153-162.

HENDERSON, S. M.; PABIS, S. Grain drying theory II. Temperature effects on drying coefficients. J. Agric. Eng. Res., v. 6, p. 169-174,1961.

MIDILLI, A.; KUCUK, H.; YAPAR, Z. A new model for single layer drying. Drying Technol; 20(7) p. 1503-13, 2002.

PAGE, G. E. Factors influencing the maximum rates of air drying shelled corn in thin layers. M. S. Thesis, Department of Mechanical Engineering, Purdue University, purdue, USA, 1949.

PARK, K. J.; BIN, A.; BROD, F. P. R. Drying of pear D'Anjou with and without osmotic dehydration. Journal of Food Engineering, v. 56, n. 1, p. 97-103, 2003.

PERRY, R. H.; GREEN, D.W. Chemical Engineers Handbook. New York: McGraw-Hill, $2150 \mathrm{p}, 1999$.

VERMA, L. R.; BUCKLIN, R. A.; ENDAN, J. B.; WRATTEN, F. T. Effects of drying air parameters on rice drying models. Trans ASAE; 28:296 - 301, 1985.

VIEIRA, R.K.; SILVEIRA, A. M. Nota Sobre o Cascateamento de Sólidos em um Secador Tambor Rotativo Considerando Apenas um Suspensor, Anais do XXIII Congresso Brasileiro de Sistemas Particulados, Vol. 1, p.403-412, Maringá - PR, 1995.

WHITE, G. M.; ROSS, I. J.; PONELERT, R. Fully exposed drying of popcorn. Trans ASAE. 24:466-8, 1981.

YAGCIOGLU, A.; DEGIRMENCIOGLU, A.; CAGATAY, F. Drying characteristic of laurel leaves under different conditions. In: Bascetincelik A, editor. Proceeding of the 7th international congress on agricultural mechanization and energy, 26 - 27 May, Adana, Turkey. Faculty of Agriculture, Cukurova Universty, p. 565-9, 1999. 